\title{
Oxidative stress and urinary system damage in fructose- induced rat model of metabolic syndrome: Effect of calorie restriction and exercise
}

\author{
Tarık Emre ŞENER 1 (D), Özge ÇEVİK 2 (D) Şule ÇETINNEL ${ }^{3}$ (D) Göksel ŞENER 4 * (D) \\ 1 Marmara University Hospital, Department of Urology, İstanbul \\ 2 Cumhuriyet University, Faculty of Pharmacy, Department of Biochemistry, Sivas \\ 3 Marmara University, School of Medicine, Department of Histology, İstanbul \\ 4 Marmara University, School of Pharmacy, Department of Pharmacology, İstanbul \\ * Corresponding Author. E-mail: gsener@marmara.edu.tr (G.Ş.); Tel. +90-216-414 2962.
}

Received: 07 February 2020 / Accepted: 26 April 2020

\begin{abstract}
The aim of this study was to investigate the effects of calorie restriction (CR) and swimming exercise (SW) on oxidative injury in kidney and bladder tissues, in rats with metabolic syndrome (MS). 3-months old rats were divided into five; Control, MS, MS+CR, MS+SW, MS+CR+SW. The metabolic syndrome model was created using $10 \%$ fructose solution in drinking water for three months. Afterwards, SW and 40\% CR were applied for six weeks. Blood glucose measurements were performed at the beginning, the third month and the end of experiment. After decapitation, blood, kidney and bladder samples were collected. Cytokine levels, antioxidant and oxidative stress parameters were examined. There was a remarkable change in blood glucose levels in MS+CR+SW group. Fructose-induced increased TNF- $\alpha$ and decreased ADP levels in plasma were reversed in CR, SW, and CR+SW groups. MDA levels were increased, while SOD and ADP levels were decreased in renal and bladder tissues in MS group. CR and SW significantly reversed all parameters in both tissues. Moreover, caspase activity increased in both tissues in MS group. CR decreased the caspase activity in kidney tissue, and in bladder tissues caspase activity significantly decreased with both CR and SW. Western blot analysis showed an increased caspase-3 protein expression in both tissues which was reversed by CR, SW, and $\mathrm{CR}+\mathrm{SW}$. The results of our study showed that MS disrupts the balance of pro/anti-inflammatory cytokines in plasma and causes oxidant damage in urinary tissues. Calorie restriction and exercise are protective against the damage caused by MS.
\end{abstract}

KEYWORDS: Metabolic syndrome; exercise; calorie restriction; kidney; bladder.

\section{INTRODUCTION}

Metabolic syndrome (MS) is an important health problem associated with important cardiovascular risk factors such as diabetes, obesity, dyslipidemia and hypertension. Oxidative stress and inflammation which play role in cellular damage and various organ dysfunctions, have a major role in MS and its clinical complications [1]. Urinary tract damage and kidney diseases are some of the clinical complications caused by MS [2].

Obesity, leading to diabetes and hypertension can initiate kidney disease through disease-associated vascular damage and oxidative stress. Although the mechanism has not been fully explained, increasing intraglomerular pressure by a compensatory hyperfiltration mechanism to meet the high metabolic requirements of increase in body weight damages the kidney tissue. However, the presence of chronic kidney disease in obese individuals without diabetes or hypertension has shown that obesity alone can also cause kidney disease [3]. The association of increased visceral adipose tissue with renal damage and even high mortality in these patients clearly emphasizes the role of visceral adiposity in organ damage [4].

On the other hand, epidemiological studies have shown that MS is also associated with BPH and bladder dysfunction. Factors such as autonomic hyperactivity and inflammation in the obese patients may play a role in these clinical conditions. The inflammation-mediated detrimental effect may be due to endocrine and inflammatory activity of adipose tissue through the production of various adipokines which eventually

How to cite this article: Şener TE, Çevik Ö, Çetinel Ö, Şener G. Oxidative stress and urinary system damage in fructose-induced rat model of metabolic syndrome: Effect of calorie restriction and exercise. J Res Pharm. 2020; 24(3): 318-325. 
cause inflammation, oxidative stress, abnormal lipid metabolism, increased insulin production and insulin resistance [2].

Since sedentary life and excessive calorie intake are responsible for many cardiovascular diseases including MS, clinicians generally prefer and offer several non-pharmacological approaches, like exercise and calorie restriction. In the light of these knowledge, in this study, the possible beneficial effects of swimming exercise and calorie restriction, and the mechanism of these effects were investigated against damage in the urinary organs induced by excessive fructose intake.

\section{RESULTS}

\subsection{Blood glucose levels}

As shown in table 1 , three months $10 \%$ fructose given in drinking water caused significant $(\mathrm{p}<0.001)$ increase in blood glucose levels in the MS, MS+CR, MS+SW and MS+CR+SW groups.

Table 1. Blood glucose levels (mg/dl) at the beginning (t1), at the end of third month (t2) and at the end of the experiment (t3). C: Control, MS: Metabolic syndrome, CR: Calorie restriction. SW: Swimming exercise.

\begin{tabular}{lcllll}
\hline Blood glucose & C & MS & MS+CR & MS+SW & MS+CR+SW \\
\hline t1 & $100 \pm 6.9$ & $92 \pm 4.1$ & $80 \pm 3.3$ & $84 \pm 3.9$ & $85 \pm 2.6$ \\
\hline t2 & $94 \pm 3.2$ & $255 \pm 13.4^{* * *}$ & $192 \pm 6.5^{* * *}$ & $181 \pm 9.6^{* * *}$ & $188 \pm 15.6^{* * *}$ \\
\hline t3 & $91 \pm 4.1$ & $249 \pm 15.3^{* * *}$ & $105 \pm 6.7^{+++}$ & $125 \pm 6.4^{*},{ }^{+++}$ & $92 \pm 3.6^{+++}$
\end{tabular}

${ }^{*} \mathrm{p}<0.05,{ }^{* * *} \mathrm{p}<0.001$ : compared with $\mathrm{t} 1$ periods in each groups.

$+++\mathrm{p}<0.001$ : compared with $\mathrm{t} 2$ periods in each groups.

\subsection{Biochemical results}

Plasma TNF-a levels were found to be significantly $(\mathrm{p}<0.01)$ due to $10 \%$ fructose in the MS group, while in the $\mathrm{CR}$ and $\mathrm{CR}+\mathrm{SW}$ group this cytokine levels were decreased. Although SW could not reduce TNF-a levels, it was also not significantly different when compared to the control. On the other hand, ADP levels that were reduced in MS group $(p<0.001)$, were found to be increased with all three, $C R(p<0.01), S W(p<0.001)$ and $C R$ $+\mathrm{SW}(\mathrm{p}<0.01)$ applications (Figure 1$)$.
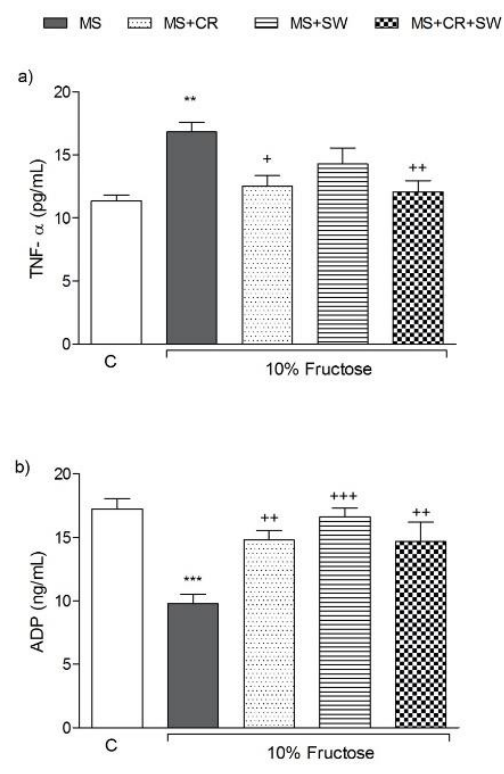

Figure 1. a) TNF-alpha and b) adiponectin (ADP) levels in plasma. MS: Metabolic syndrome, CR: Calorie restriction. SW: Swimming exercise.

${ }^{* *} \mathrm{p}<0.01,{ }^{* * *} \mathrm{p}<0.001$ : vs control group. $+\mathrm{p}<0.05,++\mathrm{p}<0.01,+++\mathrm{p}<0.001$ : vs MS group. 
The MDA levels, a lipid peroxidation index, were significantly higher $(p<0.001)$ in the MS group receiving 10\% fructose with drinking water, in both kidney and bladder tissues. In kidney CR, SW and both $C R+S W$ were effectively reduced $(p<0.001)$ the MDA levels in a similarly. In the bladder tissues CR and $\mathrm{CR}+\mathrm{SW}$ reduced the MDA ( $\mathrm{p}<0.001)$. When compared to the MS group, MDA levels in the SW group were not significantly lower and were not also significant compared to the control group. However, MDA levels of $\mathrm{SW}$ group was found to be higher $(\mathrm{p}<0.05)$ than $\mathrm{CR}$ group (Figure $2 \mathrm{a}$ and $\mathrm{b}$ ).
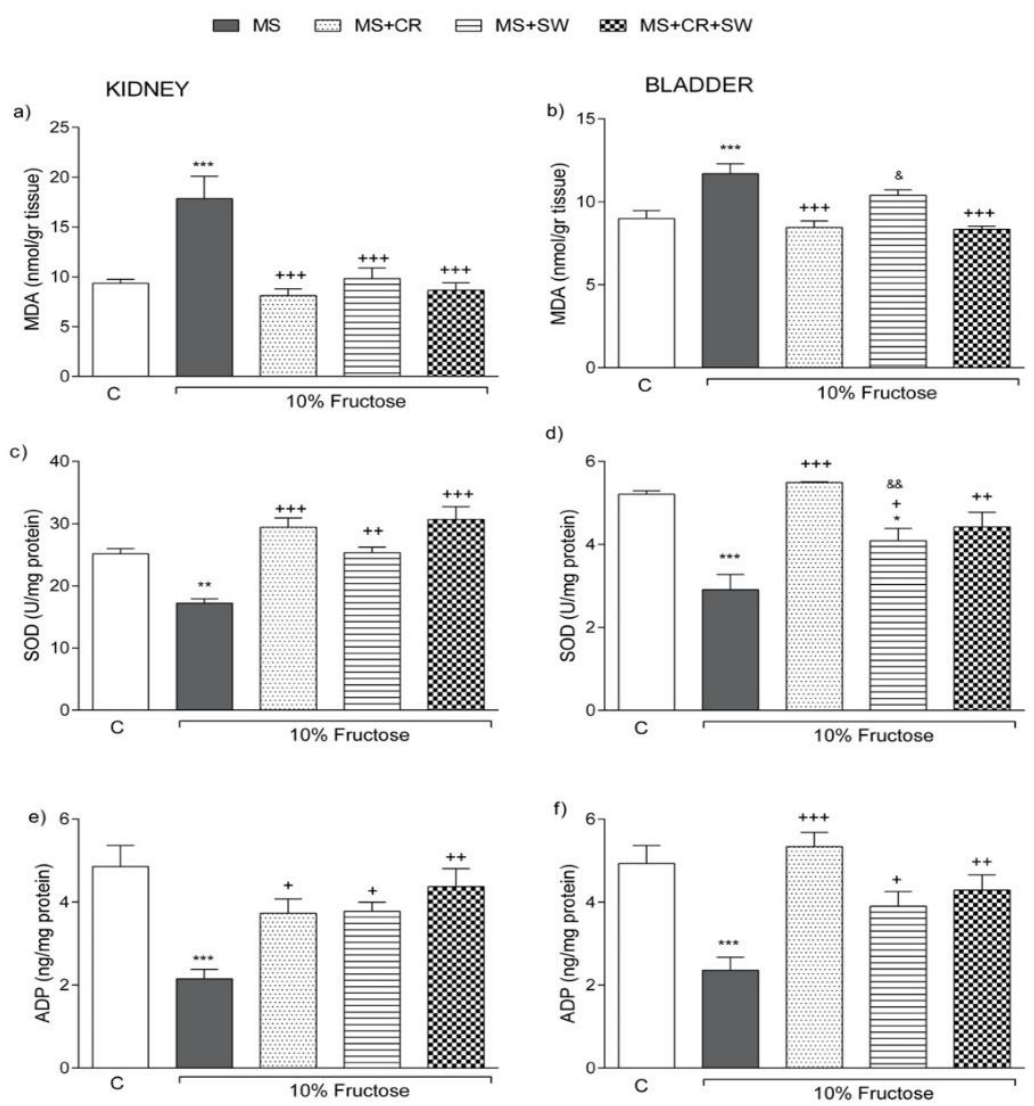

Figure 2. $a$ and b) Malondialdehyde (MDA), c and d) superoxide dismutase (SOD) and, e and f) adiponectin (ADP) levels in kidney (right column) and bladder (Left column) tissues.

MS: Metabolic syndrome, CR: Calorie restriction. SW: Swimming exercise.

${ }^{* *} \mathrm{p}<0.01,{ }^{* * *} \mathrm{p}<0.001$ : vs control group. $+\mathrm{p}<0.05,++\mathrm{p}<0.01,+++\mathrm{p}<0.001$ : vs MS group.

$\& \mathrm{p}<0.05, \& \& \mathrm{p}<0.01$ : vs $\mathrm{MS}+\mathrm{CR}$ and group.

Metabolic syndrome induced oxidative stress, as determined through increased MDA levels caused significant reduction in antioxidant enzyme SOD activity $(\mathrm{p}<0.01, \mathrm{p}<0.001)$ in both tissues. All three applications, CR, SW and CR + SW increased the enzyme activity $(\mathrm{p}<0.05-0.001)$, however there was a still decrease $(p<0.05)$ in bladder tissues in SW group compared to control, and it was also significantly lower $(\mathrm{p}<0.01)$ than $\mathrm{CR}$ group (Figure $2 \mathrm{c}$ and $\mathrm{d}$ ).

An adipocytokine, adiponectin, was found to be significantly lower $(\mathrm{p}<0.001)$ than that of the control group in both kidney and bladder tissues, while ADP levels were significantly increased $(\mathrm{p}<0.05-0.001)$, through CR, SW, and CR+SW (Figure 2e and f).

As shown in figure 3, caspase-3 activity of kidney and bladder tissues of rats with MS, were significantly higher $(\mathrm{p}<0.05, \mathrm{p}<0.001)$ than the control group. In kidney tissues only CR reduced the caspase activity $(p<0.05)$, but in bladder tissues all three treatment modalities reduced $(p<0.01)$ the enzyme activity (Figure 3a and $3 \mathrm{~b}$ ). When examined the protein expressions of Caspase-3, semi-quantitative Western blot analysis showed that caspase- 3 bands at $32 \mathrm{kDa}$, showed high intensity in both tissues $(\mathrm{p}<0.001)$. On the other hand, although protein expressions were still higher than the control $(\mathrm{p}<0.05, \mathrm{p}<0.01), \mathrm{CR}, \mathrm{SW}$, and combination of $\mathrm{CR}$ and SW were reduced these increased expressions significantly $(\mathrm{p}<0.001$, Figure $3 c-f)$. 


\subsection{Histological results}

\subsubsection{Kidney}

The control group had a regular alignment of both tubules and glomeruli (Figure 4a) whereas the MS group showed gross degeneration in the tubular and glomerular structures, interstitial edema (Figure $4 \mathrm{~b})$. In the MS+ CR group regression of interstitial edema and reduction of tubular cell desquamation was observed (Figure 4c). MS+EG group tubular cell desquamation reduced and interstitial edema was ongoing although seemed regressive (Figure $4 \mathrm{~d}$ ). Interstitial edema was slightly ongoing and both glomerular and tubular structures were regenerated (Figure 4e).
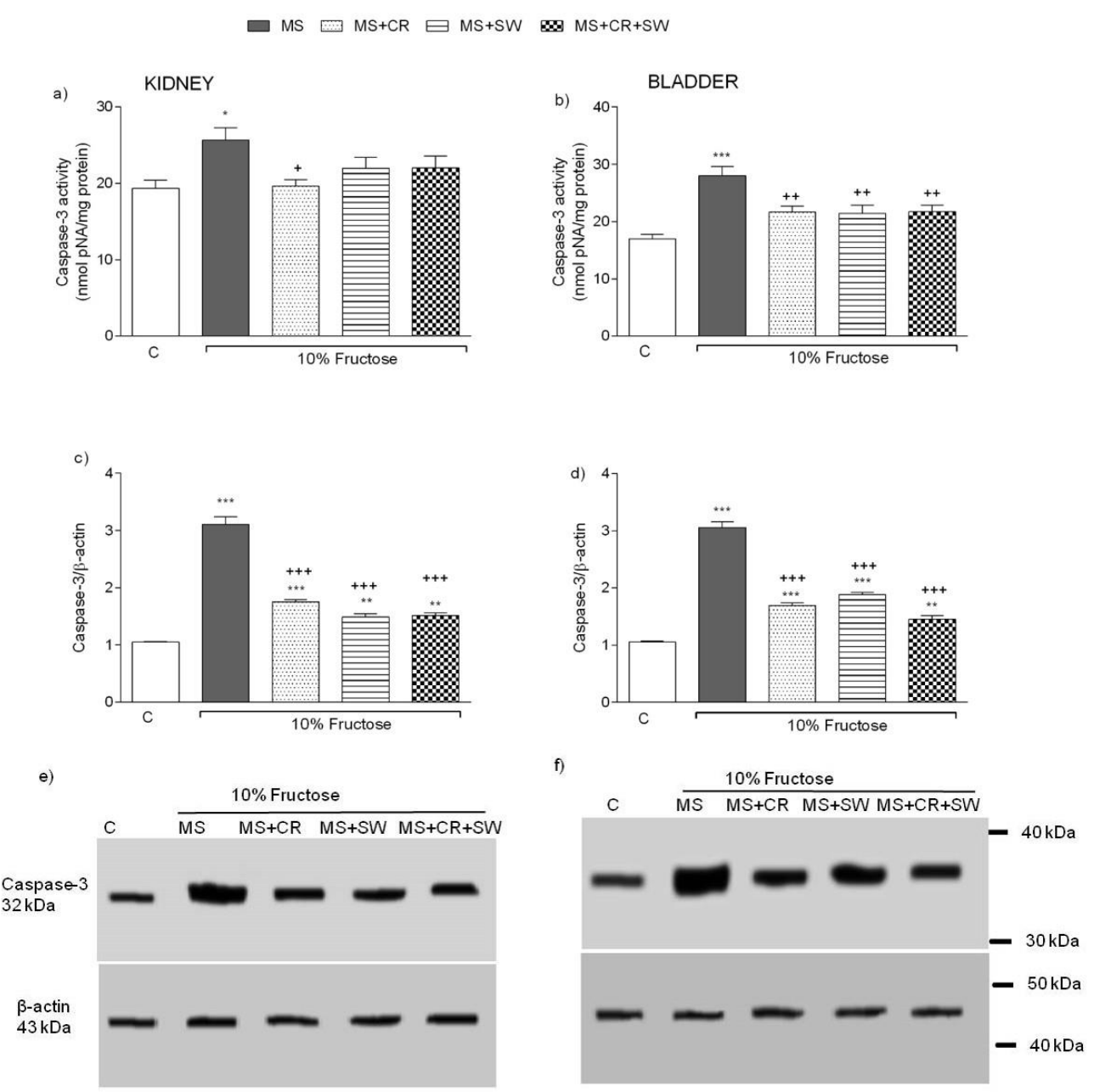

Figure 3. Caspase-3 activities in a) kidney and b) bladder tissues. Caspase-3 protein expressions in kidney (c and e) and bladder ( $\mathrm{d}$ and $\mathrm{f}$ ) tissues.

MS: Metabolic syndrome, CR: Calorie restriction. SW: Swimming exercise.

${ }^{*} \mathrm{p}<0.05,{ }^{* *} \mathrm{p}<0.01,{ }^{* * *} \mathrm{p}<0.001$ : vs control group.

$+\mathrm{p}<0.05,++\mathrm{p}<0.01,+++\mathrm{p}<0.001$ : vs MS group.

\subsubsection{Bladder}

The control bladder tissues were regularly aligned (Figure 5a) whereas in the MS group there was severe fatty edema in the muscle and lamina propria besides desquamation of epithelium (Figure $5 b$ ). In the MS+CR group the edema in the lamina propria seemed regressive (Figure 5c). MS+EG group fatty edema in the muscle tissue reduced but ongoing in the lamina propria (Figure $5 \mathrm{~d}$ ). MS+CR+EG group demonstrated prominent regression of fatty edema besides epithelial desquamation (Figure 5e). 


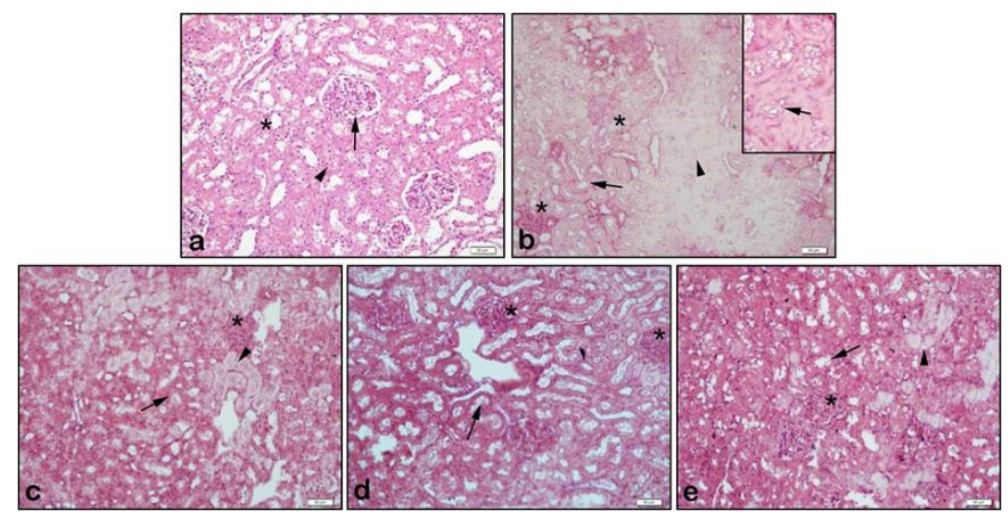

Figure 4. (a) Control group, regular morphology of both glomerulus (arrow) and tubulus (arrowhead), interstitial area $\left(^{*}\right),\left(\right.$ b) MS group, severely degenerated interstitium (arrowhead) and glomerulus $\left(^{*}\right)$, desquamation of tubular cells (arrows), (c) MS+CR group, reduction in the interstitial edema (arrowhead), slight regeneration in the tubular (arrow) and glomerulus (*) structure, (d) MS+EG group, tubular cellularity maintained (arrow) and so the glomeruli $(*)$, (e) MS+CR+EG group, regression of the degeneration of tubules (arrow) and glomeruli $\left({ }^{*}\right)$ some focal tubular areas showed slight degeneration (arrowhead). HE staining.

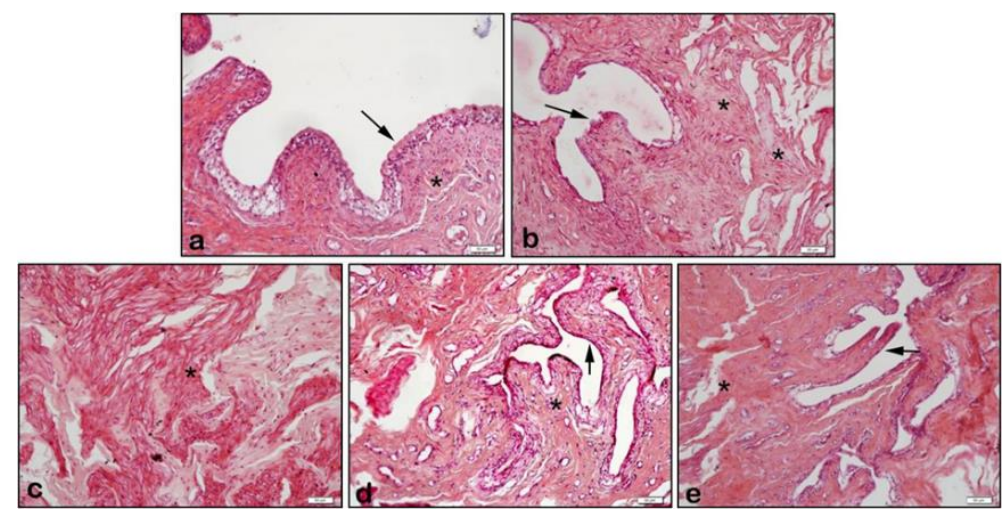

Figure 5. (a) Control group, regular alignment of bladder epithelium (arrow) and lamina propria (*), (b) MS group, desquamated epithelium (arrow) and fatty edema in muscle and lamina propria (*), (c) MS+CR group, regression of fatty edema in muscle tissue $\left(^{*}\right)$ although ongoing in the lamina propria, (d) MS+EG group, regression of fatty edema in the muscle tissue $\left(^{*}\right)$ and regenerated epithelium (arrow), (e) MS+CR+EG group, epithelium gained its integrity (arrow) and reduced edema in both mucle $\left(^{*}\right)$ and lamina propria.

\section{DISCUSSION}

Metabolic syndrome (MS) is characterized by the presence of hyperinsulinemia or insulin resistance where some vascular risk factors such as dyslipidemia, hypertension or central obesity are accompanied. Furthermore, hypercoagulability, endothelial dysfunction and inflammation were also seen in these same individuals $[5,6]$. Increasing evidence supports the idea that these key components of MS are also risk factors for urinary tract diseases and thus there is a link between MS and many common urological conditions [7, 8]. On the other hand it is well known that oxidative stress plays an essential role in these individual components of MS [9]. Accordingly in this study we have investigated the antioxidative and tissue protective effects of calorie restriction and swimming exercise, on the urinary system of the rats with experimentally induced metabolic syndrome

It has been observed that the increase in visceral adipose tissue above normal causes many disturbances in lipoprotein metabolism [10]. This causes an increase in intraabdominal fatty tissue, insulin resistance, diabetes and cardiovascular diseases and eventually MS. In addition, adipose tissue, which secretes bioactive adipocytokines such as TNF- $\alpha$ and adiponectin (ADP), which has a central role in the development of insulin resistance, has a major role in the metabolic syndrome [11].

Environmental factors such as excessive feeding or immobility lead to an increase in visceral fatty tissues and a consequent decrease in plasma adiponectin levels. It has been demonstrated that the decrease in adiponectin levels can cause oxidative stress, diabetes mellitus, hypertension and dyslipidemia [11]. In our 
study, in rats subjected to high-fructose diet, plasma TNF-a levels increased while a decrease in ADP levels was observed. In the study by Rodriguez et al., it has been shown that restoration of insulin sensitivity with diet and exercise had lead to a regulation of blood pressure and a decrease in lipid and inflammatory cytokine levels [12]. Similar to these studies, our study also showed that the increase in TNF- $\alpha$ and the decrease in ADP due to MS were prevented with both calorie restriction and exercise.

The relation between MS and inflammation has previously been proved with the increase in proinflammatory cytokines and c-reactive protein, which is a nonspecific marker of inflammation. Obesity, while causing an increase in adipose tissue, also causes a release of chemokines and consequently macrophages infiltrate the tissues and start the inflammatory process. On the other hand, this inflammatory process causes an increase in free radical production. Ultimately, the relation between metabolic syndrome and oxidative tissue damage can help us understand both the pathophysiology of this syndrome and also the effects on remote tissues. Another marker that is widely used to determine oxidative damage is MDA, which is also known as thiobarbituric acid reactive substances (TBARs) and is a final product of lipid peroxidation. In our study, the increased MDA levels in both kidney and bladder tissues in rats with MS proves the presence of oxidative stress in these tissues. Another marker of inflammation is SOD, whose activity is inversely correlated with MDA levels. SOD activity, as a marker of antioxidant activity, decreases due to depletion in cases of inflammation. In literature, it has been shown that acute exercise could increase oxidative stress but however, it has also been shown that regular committed exercise could cause a decrease in the oxidative parameters caused by acute exercise and could also trigger the production of antioxidants [13]. Therefore the authors recommend that regular committed exercise should be undertaken as a regular life-style modification recommendation [13].

Regular exercise increases the antioxidant enzyme activities such as SOD and GSH activities and therefore prevents the unwanted effects of oxidative stress [14]. Regular low/moderate-intensity exercise has also been proven to provide a significant improvement (recovery) in glucose intolerance and decrease obesityassociated organ dysfunctions [14].

Calorie restriction, an important lifestyle modification requirement apart from regular exercise, has been recommended to combat the aging and aging dependent neurodegenerative and cardiovascular diseases. The application of calorie restriction plays an important role not only in weight control but also in the fight against oxidative tissue damage.

It is well known that changes in the redox status increase oxidative stress and trigger caspase activation and consequently, cells are destroyed either by necrosis or apoptosis. ROS can induce apoptosis by cleavage of poly (ADPribose) polymerase (PARP), increasing mitochondrial permeability and thus release of cytochrome C, and activating caspases. Since apoptosis is related to oxidative events and oxidative stress is an important process due to MS, it is reasonable that the increase in MDA caused by MS can lead to caspase activation [15]. In our study MS induction caused a significant increase in oxidative injury determined through the increase in MDA levels, and the decreases in antioxidant enzyme activities and triggered caspase activation and expression.

\section{CONCLUSION}

Metabolic syndrome is a world-wide important health problem with various concomitant organ dysfunctions due oxidative stress and inflammation. In this study, urinary system tissue damage caused by MS was demonstrated with increased oxidative stress biomarkers and decreased antioxidant enzyme levels in kidney and bladder tissues. Also, in this study, calorie restriction and exercise were proven to decrease the oxidative stress and provide tissue protection against MS-induced inflammatory process.

With further clinical studies, these important lifestyle modifications such as calorie restriction and exercise, can be implemented in treatment strategies of metabolic syndrome to prevent urinary system tissue damage.

\section{MATERIALS AND METHODS}

\subsection{Animals and ethics}

Male Sprague-Dawley rats weighing 250-300 grams were used for the experiments. Animals were supplied from the animal facility at Marmara University (MU) Experimental Animal Implementation and Research Centre. All experimental protocols were performed according to the MU Animal Care and Use Committee (Protocol number: 82-2.2012.mar). 
Animals were randomly divided into five groups (10 rats in each). Control (C), metabolic syndrome (MS), MS+ calorie restriction (CR), MS + swimming exercise (SW), and MS+CR+SW groups.

\subsection{Metabolic syndrome}

Metabolic syndrome was induced by giving drinking water to rats containing $10 \%$ fructose (wt/vol) for 12 weeks. For calorie restriction, after determining the daily food intake of the animals, daily food intake was limited to $40 \%$ for 6 weeks. In the exercise groups, rats were subjected to moderate swimming exercise. For this purpose, the rats were floated in the heated water pool for 30 minutes a day, 3 days a week during the 6week period during which they exercised.

\subsection{Blood glucose levels}

At the beginning of the experiment, at the end of the 3rd month and at the end of the experiment blood glucose levels were measured using a glucometer.

\subsection{Biochemical analysis}

After decapitation, blood and tissue (kidney and bladder) samples were taken. Blood samples were analyzed for adiponectin (ADP) and TNF-a levels while oxidative stress indicators, malondialdehyde (MDA) and ADP levels and superoxide dismutase (SOD) and catalase-3 activities were examined in tissue samples. Tissues were also examined for morphological evaluation.

Serum and tissue adiponectin (ADP) levels were determined according to the kit procedure using the commercial kit (sc-26496, Santa Cruz), and TNF-a levels were quantified using enzyme-linked immunosorbent assay (ELISA) kits (Biosource Europe S.A. Nivelles, Belgium).

The MDA levels in kidney and bladder tissues were assayed as a product of lipid peroxidation through monitoring thiobarbituric acid reactive substance formation. Results are expressed as nmol MDA/g tissue [16].

Superoxide dismutase (SOD) activity in tissue samples was measured according to the method described by Mylroie et al. [17]. A standard curve was prepared routinely with bovine SOD (Sigma Chemical Co, Saint Louis, USA; S-2515, $3000 \mathrm{U}$ ) as reference. Absorbance readings were taken at 0 and $8 \mathrm{~min}$ of illumination and net absorbances were calculated.

Caspase- 3 activity assay was performed using the caspase- 3 cellular activity assay kit (Calbiochem, San Diego, CA) according to the manufacturer's instructions. The DEVDpNA cleavage activity was calculated as $\mathrm{pmol} / \mathrm{min} / \mathrm{mg}$ protein. Protein concentration in tissue samples was determined using Bradford method [18].

Caspase-3 protein expression was measured directly by Western blot using a monoclonal rat anticaspase-3 and cleaved caspase-3 (clv-cas3) antibodies. Samples were homogenized with cell lysis buffer and then centrifuged sample supernatant was collected for determining immunobloting assay. Samples resolved on $12 \%$ SDS-PAGE and transferred to nitrocellulose membrane. The membrane was blocked with 5\% nonfat skimmed milk powder then washed with TBST and incubated overnight with primary antibody (1:500 monoclonal rat anti-caspase-3 sc-56055; anti-b-actin, sc-47778 from Santa Cruz Biotechnology, clv-casp3 9664 from Cell Signaling). The membrane was incubated with HRP conjugated secondary antibody for $2 \mathrm{~h}$. After the incubation, membrane was developed with chemiluminescence reagents (sc-2048, Santa Cruz Biotechnology L kit) and exposed to Fuji Super RX fi lm (47410, Tokyo, Japan). Protein bands were analyzed using "Image J Programme Optical Density Analysis Software" (NIH, USA). Signals were normalized with respect asdft to $\beta$-actin.

\subsection{Histological analysis}

Tissues were fixed in 10\% formalin solution and dehydrated in degraded ethanol series and cleared in toluene. Paraffin-embedded samples were cut with $5 \mu \mathrm{m}$ thickness by rotary microtome and stained with hematoxylinand-eosin (H\&E). Sections were evaluated and photographed under Olympus BX51 light microscope (Olympus Co., Ltd., Tokyo, Japan).

\subsection{Statistical analysis}

Statistical analysis was performed using GraphPad Prism 5.0 (GraphPad Software, San Diego, CA, USA). Each group consisted of six animals. All data are expressed as the mean \pm SEM. Groups of data were compared with ANOVA followed by Turkey's multiple comparison tests. Statistical significance was accepted as $\mathrm{p}<0.05$. 


\section{Acknowledgements:}

Author contributions: Concept - Ş.T.E., Ş.G.; Design - Ş.T.E., Ş.G., Ç.Ş.; Supervision - Ş.G., Ç.S..; Resources - Ş.T.E., Ç.Ö., Ş.G., Ç.Ş.; Materials - Ş.T:E., Ç.Ö., Ş.G., Ç.Ş.; Data Collection and/or Processing - Ş.T.E., Ç.Ö., Ş.G., Ç.Ş.; Analysis and/or Interpretation -Ş.T.E., Ç.Ö., Ş.G., Ç.Ş.; Literature Search -Ş.T.E., Ç.Ö., Ş.G., Ç.Ş.; Writing - Ş.T.E., Ç.Ö., Ş.G., Ç.Ş.; Critical Reviews - Ş.T:E., Ç.Ö., Ç.Ş., Ş.G.

Conflict of interest statement: The authors declare that they have no conflict of interest.

Ethics committee approval: All experimental protocols were performed according to the MU Animal Care and Use Committee (Protocol number: 82-2.2012.mar).

\section{REFERENCES}

[1] Spahis S, Borys JM, Levy E. Metabolic Syndrome as a Multifaceted Risk Factor for Oxidative Stress. Antioxid Redox Signal. 2017; 26(9): 445-61. [CrossRef]

[2] Kovesdy CP, Furth S, Zoccali C, World Kidney Day Steering C. Obesity and kidney disease: Hidden consequences of the epidemic. Indian J Nephrol. 2017; 27(2): 85-92. [CrossRef]

[3] Chang Y, Ryu S, Choi Y, Zhang Y, Cho J, Kwon MJ, Hyun YY, Lee KB, Kim H, Jung HS, Yun KE, Ahn J, Rampal S, Zhao D, Suh BS, Chung EC, Shin H, Pastor-Barriuso R, Guallar E. Metabolically Healthy Obesity and Development of Chronic Kidney Disease: A Cohort Study. Ann Intern Med. 2016; 164(5): 305-312. [CrossRef]

[4] La Russa D, Giordano F, Marrone A, Parafati M, Janda E, Pellegrino D. Oxidative Imbalance and Kidney Damage in Cafeteria Diet-Induced Rat Model of Metabolic Syndrome: Effect of Bergamot Polyphenolic Fraction. Antioxidants (Basel). 2019; 8(3). [CrossRef]

[5] Buege JA, Aust SD. Microsomal lipid peroxidation. Methods Enzymol. 1978; 52: 302-310. [CrossRef]

[6] Mylroie AA, Collins H, Umbles C, Kyle J. Erythrocyte superoxide dismutase activity and other parameters of copper status in rats ingesting lead acetate. Toxicol Appl Pharmacol. 1986; 82(3): 512-520. [CrossRef]

[7] Bradford MM. A rapid and sensitive method for the quantitation of microgram quantities of protein utilizing the principle of protein-dye binding. Anal Biochem. 1976; 72: 248-254. [CrossRef]

[8] Guize L, Pannier B, Thomas F, Bean K, Jego B, Benetos A. Recent advances in metabolic syndrome and cardiovascular disease. Arch Cardiovasc Dis. 2008; 101(9): 577-583. [CrossRef]

[9] Huang PL. eNOS, metabolic syndrome and cardiovascular disease. Trends Endocrinol Metab. 2009; 20(6): 295-302. [CrossRef]

[10] Gorbachinsky I, Akpinar H, Assimos DG. Metabolic syndrome and urologic diseases. Rev Urol. 2010; 12 (4): e157-80.

[11] He Q, Wang Z, Liu G, Daneshgari F, MacLennan GT, Gupta S. Metabolic syndrome, inflammation and lower urinary tract symptoms: possible translational links. Prostate Cancer Prostatic Dis. 2016; 19(1): 7-13. [CrossRef]

[12] McCracken E, Monaghan M, Sreenivasan S. Pathophysiology of the metabolic syndrome. Clin Dermatol. 2018; 36(1): 14-20. [CrossRef]

[13] Pouliot MC, Despres JP, Lemieux S, Moorjani S, Bouchard C, Tremblay A, et al. Waist circumference and abdominal sagittal diameter: best simple anthropometric indexes of abdominal visceral adipose tissue accumulation and related cardiovascular risk in men and women. Am J Cardiol. 1994; 73(7): 460-468. [CrossRef]

[14] Okamoto Y. Adiponectin provides cardiovascular protection in metabolic syndrome. Cardiol Res Pract. 2011; 2011: 313179. [CrossRef]

[15] Rodriguez A, Ezquerro S, Mendez-Gimenez L, Becerril S, Fruhbeck G. Revisiting the adipocyte: a model for integration of cytokine signaling in the regulation of energy metabolism. Am J Physiol Endocrinol Metab. 2015; 309(8): E691-714. [CrossRef]

[16] Clarkson PM. Antioxidants and physical performance. Crit Rev Food Sci Nutr. 1995; 35(1-2): 131-41. [CrossRef]

[17] Botezelli JD, Cambri LT, Ghezzi AC, Dalia RA, PP MS, Ribeiro C, et al. Different exercise protocols improve metabolic syndrome markers, tissue triglycerides content and antioxidant status in rats. Diabetol Metab Syndr. 2011 ; 3: 35. [CrossRef]

[18] Kannan K, Jain SK. Oxidative stress and apoptosis. Pathophysiology. 2000; 7(3): 153-63. [CrossRef]

This is an open access article which is publicly available on our journal's website under Institutional Repository at http://dspace.marmara.edu.tr. 